\title{
The Temperature Structure of the Warm-Hot Intergalactic Medium
}

\section{Citation}

Yoshida, Naoki, Steven R. Furlanetto, and Lars Hernquist. 2004. "The Temperature Structure of the Warm-Hot Intergalactic Medium." The Astrophysical Journal 618 (2): L91-94. https:// doi.org/10.1086/427874.

\section{Permanent link}

http://nrs.harvard.edu/urn-3:HUL.InstRepos:41381769

\section{Terms of Use}

This article was downloaded from Harvard University's DASH repository, and is made available under the terms and conditions applicable to Other Posted Material, as set forth at http:// nrs.harvard.edu/urn-3:HUL.InstRepos:dash.current.terms-of-use\#LAA

\section{Share Your Story}

The Harvard community has made this article openly available.

Please share how this access benefits you. Submit a story.

Accessibility 


\title{
THE TEMPERATURE STRUCTURE OF THE WARM-HOT INTERGALACTIC MEDIUM
}

\author{
NaOki Yoshida ${ }^{1}$, Steven R. Furlanetto ${ }^{2}$, and Lars Hernquist ${ }^{3}$ \\ Submitted to ApJ Letters
}

\begin{abstract}
We study the temperature structure of the intergalactic medium (IGM) using a large cosmological $N$ body/SPH simulation. We employ a two-temperature model for the thermal evolution of the ionized gas, in which we include explicitly the relaxation process between electrons and ions. In the diffuse, hot IGM, the relaxation time is comparable to the age of the Universe and hence the electron temperature in postshock regions remains significantly smaller than the ion temperature. We show that, at the present epoch, a large fraction of the warm/hot intergalactic medium (WHIM) has a well-developed two-temperature structure, with typical temperature differences of order a few. Consequently, the fraction of metals in various ionization states such as OvI, OvII, and OvIII, as well as their line emissivities, can differ locally by more than an order of magnitude from those computed with a single-temperature model, especially in gas with $T \sim 10^{7} \mathrm{~K}$. It is thus necessary to follow the evolution of the electron temperature explicitly to determine absorption and emission by the WHIM. Although equipartition is nearly achieved in the denser intracluster medium (ICM), we find an appreciable systematic deviation between the gas-mass weighted electron temperature and the mean temperature even at half the virial radii of clusters. There is thus a reservoir of warm $\left(T_{e}<1 \mathrm{keV}\right)$ gas in and around massive clusters. Our results imply that relaxation processes need to be considered in describing and interpreting observational data from existing X-ray telescopes as well as from future missions designed to detect the WHIM, such as the Diffuse Intergalactic Oxygen Surveyor and the Missing Baryon Explorer.
\end{abstract}

Subject headings: cosmology: theory - intergalactic medium

\section{INTRODUCTION}

The distribution of baryons in the Universe remains one of the puzzling issues in modern cosmology. At the present epoch, the total amount of baryons inferred from a census of Hi absorption, gas and stars in galaxies, and X-ray emission from hot gas in galaxy clusters is far smaller than that predicted by nucleosynthesis calculations (Persic \& Salucci 1992; Fukugita, Hogan \& Peebles 1998) and by measurements of the cosmic microwave background radiation (Spergel et al. 2003). Hence, it is believed that a large fraction of the baryons lies in an as yet undiscovered dark state.

Three dimensional hydrodynamic simulations of cosmic structure formation (e.g., Cen \& Ostriker 1999; Davé et al. 2001; Croft et al. 2002) predict that about 30-40\% of the baryons reside in the so-called warm-hot intergalactic medium (WHIM) at the present epoch. This gas is mostly shock-heated to a temperature of $\sim 10^{5}-10^{7} \mathrm{~K}$ during large scale structure formation, and this relatively low temperature makes its thermal emission difficult to detect with conventional X-ray probes.

A variety of observational approaches have been considered for studying the WHIM, including both ultraviolet (e.g., Furlanetto et al. 2003, 2004) and X-ray lines. With respect to the latter possibility, there have been several tentative claims that the WHIM has been detected locally in absorption (e.g., Nicastro et al. 2002) and in emission (Finoguenov et al. 2003). Moreover, Yoshikawa et al. (2004) have proposed using OviI/OviII lines to examine the WHIM in detail with high spectral resolution X-ray detectors. They argue that, under a variety of assumptions, about half of the WHIM (by mass) can be detected via oxygen line emission (see also Fang et al. 2004). While the proposed missions appear promising, the detectability depends crucially on the assumed metallicity and the relative abundances of particular ions.

Previous theoretical studies used a single-temperature model for the WHIM, under the assumption that equipartition is fully achieved. However, the outskirts of galaxy clusters may actually have a two-temperature structure (e.g., Fox \& Loeb 1997; Takizawa 1998; Courty \& Alimi 2004). Near galaxy clusters, infalling gas shock-heats to roughly the virial temperature as kinetic energy is converted into thermal energy. Because the infalling ions carry nearly all of the bulk kinetic energy (exceeding that contained in electrons by a factor $\sim m_{p} / m_{e}$ ), the postshock electrons can gain thermal energy only via collisions with ions. If collisional relaxation takes a time comparable to or longer than the age of the Universe, the two temperatures, i.e. those of the electrons and ions, remain different. One can easily show that the relaxation time is rather long for diffuse warm-hot gas around clusters and in large-scale filamentary structures. Since the relative abundance of metal ions and their emissivities are sensitive to the electron temperature, this has important implications for future observations of the WHIM, particularly for the hotter component.

In this Letter, we study the thermal evolution of the intergalactic medium (IGM) using a large hydrodynamic

\footnotetext{
${ }^{1}$ Department of Physics, Nagoya-University, Furocho, Chikusa, Nagoya 464-6082, Japan

${ }^{2}$ Mail Code 130-33; California Institute of Technology; Pasadena, CA 91125

${ }^{3}$ Harvard-Smithsonian Center for Astrophysics, 60 Garden Street, Cambridge, MA 02138
} 


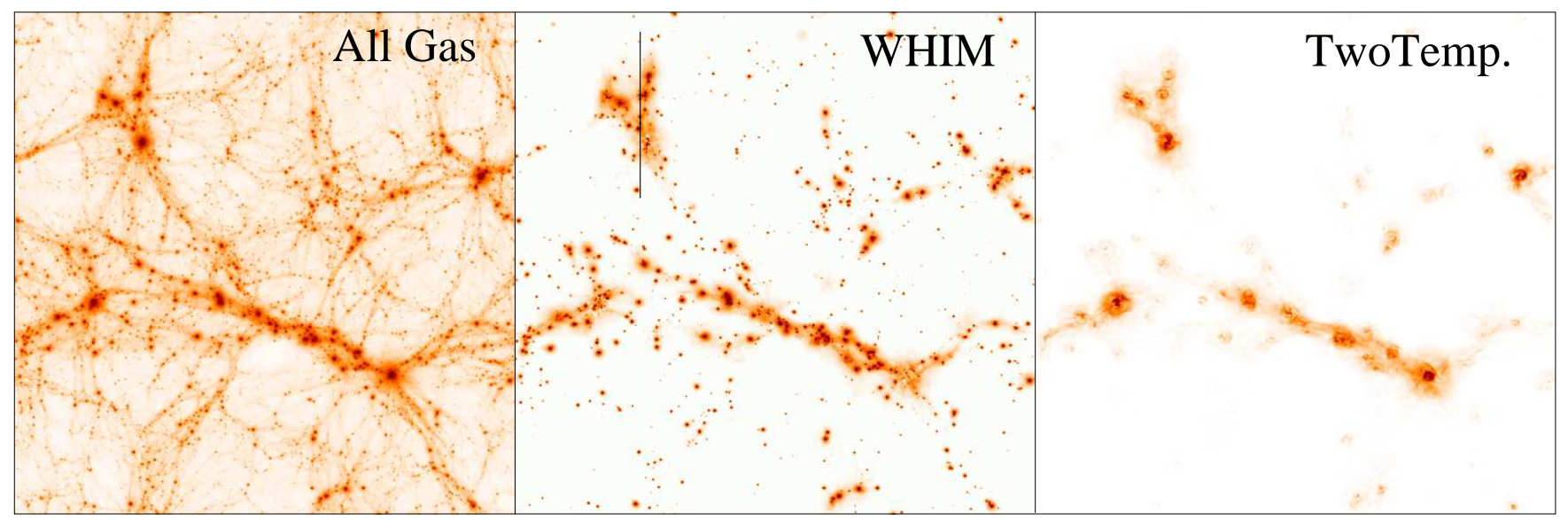

Fig. 1. - The distribution of baryons (left), the warm/hot component with $10^{6}<T<10^{7} \mathrm{~K}$ (middle), and gas with $T_{\mathrm{e}}<0.5 T_{\mathrm{i}}$ (right) in a slab of $100 \times 100 \times 20\left(h^{-1} \mathrm{Mpc}\right)^{3}$ at $z=0$. The vertical bar in the middle panel indicates a 'super-cluster' region, for which we compute the soft X-ray intensity (see section 4 and Figure 5).

simulation of structure formation. We show that a considerable fraction of the WHIM indeed has a two-temperature structure, particularly in and around rich clusters. In these regions, the electron temperature is typically smaller than the ion temperature by a factor of a few, substantially modifying estimates of the abundances of ions and their emissivities.

\section{THE $N$-BODY/SPH SIMULATIONS}

For our simulations, we use the parallel Tree-PM/SPH code GADGET2 (V. Springel, in preparation), which employs a fully conservative scheme for integrating the equations of motion (Springel \& Hernquist 2002). We implement non-equipartition processes between ions and electrons following Fox \& Loeb (1997) and Takizawa (1998). Equilibrium in an electron-proton plasma is achieved in the following manner. After passing through a shock, electrons and ions thermalize into (separate) Maxwellian distributions on equilibration timescales $t_{\mathrm{ee}}, t_{\mathrm{ii}}$, with $t_{\mathrm{ii}} / t_{\mathrm{ee}} \sim$ $\left(m_{\mathrm{i}} / m_{\mathrm{e}}\right)^{1 / 2} \sim 43$ (Spitzer 1962). Here, $m_{\mathrm{e}}$ and $m_{i}$ are the electron and ion masses and we have assumed protons dominate the ionic component. Equipartition between protons and electrons is achieved on an even longer timescale $t_{\mathrm{ei}} \sim\left(m_{\mathrm{i}} / m_{\mathrm{e}}\right) t_{\mathrm{ee}}$. Hence, we assume that electrons and ions quickly achieve Maxwellian distributions with temperatures $T_{\mathrm{e}}$ and $T_{\mathrm{i}}$, respectively, and consider only the non-equipartition effect. Spitzer (1962) showed that the appropriate timescale is given by

$$
t_{\mathrm{ei}}=\frac{3 m_{\mathrm{e}} m_{\mathrm{i}}}{8(2 \pi)^{1 / 2} n_{i} Z_{i}^{2} e^{4} \ln \Lambda}\left(\frac{k T_{\mathrm{e}}}{m_{\mathrm{e}}}+\frac{k T_{i}}{m_{i}}\right)^{3 / 2},
$$

where $e$ is the electric charge, $Z_{\mathrm{i}}$ is the charge of an ion, $n_{\mathrm{i}}$ is the ion number density, and $\ln \Lambda$ is the Coulomb logarithm, which is given by

$$
\ln \Lambda=37.8+\ln \left(\frac{T_{e}}{10^{7} \mathrm{~K}}\right)-\frac{1}{2} \ln \left(\frac{n}{10^{-5} \mathrm{~cm}^{-3}}\right) .
$$

For a hot, fully ionized plasma,

$$
t_{\mathrm{ei}}=6.3 \times 10^{8} \mathrm{yr} \frac{\left(T_{\mathrm{e}} / 10^{7} \mathrm{~K}\right)^{3 / 2}}{\left(n / 10^{-5} \mathrm{~cm}^{-3}\right)(\ln \Lambda / 40)},
$$

and this timescale can be comparable to the Hubble time in regions with $T \gtrsim 10^{7} \mathrm{~K}$ and overdensities $10-100$ with respect to the cosmic mean. The evolution of the electron temperature is given by

$$
\frac{d T_{\mathrm{e}}}{d t}=\frac{T_{\mathrm{i}}-T_{\mathrm{e}}}{t_{\mathrm{ei}}}+(\gamma-1) \frac{T_{\mathrm{e}}}{n} \frac{d n}{d t} .
$$

We do not consider heat conduction on the assumption that it will be suppressed by tangled magnetic fields. Some other electron heating processes driven by plasma instabilities have also been proposed (Laming 2000), but detailed calculations in the context of supernova remnants suggest that these mechanisms convert less than $20 \%$ of the bulk kinetic energy to electron thermal energy (e.g., Cargill \& Papadopoulos 1988), so we neglect these effects. Our treatment therefore maximizes the possible offset between $T_{\mathrm{e}}$ and $T_{\mathrm{i}}$.

We work with a flat $\Lambda$-dominated cold dark matter cosmology with matter density $\Omega_{0}=0.3$, cosmological constant $\Omega_{\Lambda}=0.7$ and expansion rate at the present time $H_{0}=70 \mathrm{~km} \mathrm{~s}^{-1} \mathrm{Mpc}^{-1}$. We set the baryon density $\Omega_{\mathrm{b}}=0.04$ and the normalization parameter $\sigma_{8}=0.9$. Our simulation employs $324^{3}$ cold dark matter particles and the same number of non-radiative gas particles in a cosmological volume $100 h^{-1} \mathrm{Mpc}$ on a side. The mass per gas particle is $3.26 \times 10^{8} h^{-1} M_{\odot}$, and the nominal gas mass resolution is about $10^{10} h^{-1} M_{\odot}$ for our choice of the number of SPH neighbors, $N=32$. We note that the CDM initial conditions and the large simulation volume allow us to correctly model the merging process during the formation of filamentary structures and the assembly of massive halos which contribute to heating of the IGM as well as the intracluster medium (ICM; e.g., Keshet et al. 2003).

\section{RESULTS}

Figure 1 shows the baryon distribution at $z=0$ in a slice $20 h^{-1}$ Mpc thick (top), the hot component with temperature $10^{6}<T<10^{7} \mathrm{~K}$ (middle), and gas in which $T_{\mathrm{e}}<0.5 T_{\mathrm{i}}$. The latter two components are quite similar, suggesting that much of the hotter WHIM has a two-temperature structure. The distribution of the twotemperature gas appears quite complex even in clusters, 


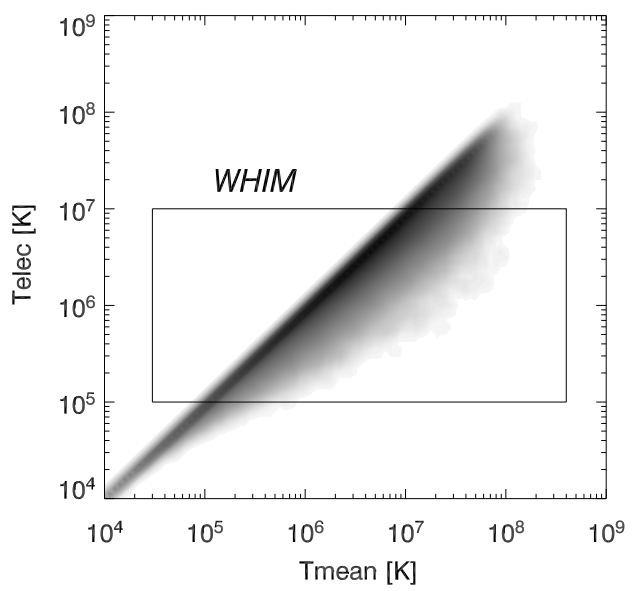

FIG. 2.- Electron temperature plotted against the mean gas temperature. The number density of gas particles is shown in greyscale. The rectangular box indicates the so-called WHIM which we define to have $10^{5}<T_{\mathrm{e}}<10^{7} \mathrm{~K}$.

indicating that shocks induced by mergers during hierarchical assembly propagate and leave two-temperature gas within clusters. We have checked the time variation of the specific entropy of the gas elements and verified that the two-temperature regions have recently been shockheated. In the gas around large-scale structure, continuous shock-heating disturbs equipartition sufficiently close to the shock, while more distant gas is often relaxed.

Figure 2 shows the electron temperature $T_{\mathrm{e}}$ plotted against the mean temperature $\bar{T}=\left(n_{\mathrm{e}} T_{\mathrm{e}}+n_{\mathrm{i}} T_{\mathrm{i}}\right) /\left(n_{\mathrm{e}}+n_{\mathrm{i}}\right)$, which is the temperature we would obtain in a single temperature model. Interestingly, the two-temperature deviation is largest in the WHIM regime (indicated by the rectangular box). Note that there are some gas elements with $T_{\mathrm{e}} \ll T_{\mathrm{i}}$. This is because our simulation does not include electron heating processes other than Coulomb collisions.

Figure 3 shows the radial distribution of $T_{\mathrm{e}}$ (solid line) and $\bar{T}$ (dashed line) for one of the most massive halos in our simulation. We used a friends-of-friends group-finder to locate dark matter halos and define the virial radius such that the mean matter density within this radius is 200 times the critical density. The halo we consider here has a virial mass of $3 \times 10^{14} h^{-1} \mathrm{M}_{\odot}$ and a virial radius of $1.1 h^{-1}$ Mpc. In Figure 3, the difference between the two temperatures is large at $r>1 h^{-1} \mathrm{Mpc}$ and appreciable even at $r<1 h^{-1} \mathrm{Mpc}$. The overall profile looks similar to those found in simulations of single clusters by Takizawa (1998) and Chieze et al. (1998). Owing to the difference in relaxation time (see eq. [3]) in the dense central part and in the low density outer regions, the electron temperature profile appears steeper than that of the mean temperature at $r>0.1 h^{-1}$ Mpc. The bottom panel of Figure 3 shows the baryonic mass fraction as a function of both $T_{\mathrm{e}}$ and $\bar{T}$ (thick and thin histograms, respectively). We selected gas particles within $5 h^{-1} \mathrm{Mpc}$ of the cluster center. The distribution of $T_{e}$ is skewed significantly to smaller values. The total gas mass contained in this region $\left(r<5 h^{-1} \mathrm{Mpc}\right)$ is $1.0 \times 10^{14} h^{-1} \mathrm{M}_{\odot}$, and nearly $50 \%$ of it has $T_{\mathrm{e}}<1 \mathrm{keV}$; i.e., the mass of the warm
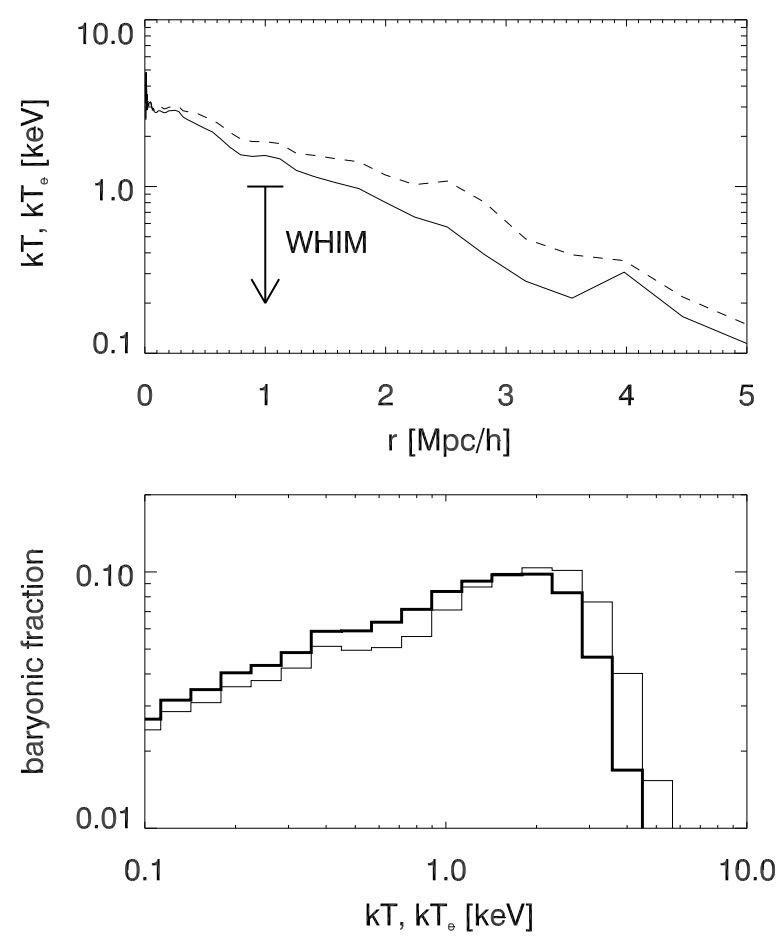

FIG. 3.- Radial distribution of the electron temperature (solid line) and the mean temperature (dashed line) in one of the most massive clusters in our simulation (top panel). Bottom panel shows the mass fraction of gas within $5 h^{-1} \mathrm{Mpc}$ from the cluster center in temperature bins, using the electron temperature (thick histograms) and the mean temperature (thin histograms).

component surrounding the cluster is greater than the gas mass within the cluster itself.

\section{COSMOLOGICAL IMPLICATIONS}

The two-temperature structure of the WHIM and ICM has many important implications. For example, the relative fractions of metal ions are of considerable interest for WHIM observations. Figure 4 shows the fraction of Ovi, OviI, and OviII ions as a function of temperature assuming collisional ionization equilibrium. ${ }^{4}$ Clearly the abundances have a strong dependence on electron temperature, because the populations are primarily determined by electron collisions (photo-ionization is unimportant for these high-level ions unless $T \ll 10^{5.5} \mathrm{~K}$ or the incident $\mathrm{X}$-ray intensity is high). The bottom panel indicates the possible errors caused by assuming a single temperature for the WHIM. A factor of two systematic shift in temperature, typical of the offsets we find between $\bar{T}$ and $T_{\mathrm{e}}$ near shocks, can lead to significant over/under-estimates of the abundances.

We further quantify the importance of non-equipartition with a model oxygen line emission map. We put the supercluster region indicated in Figure 1 at $z=0.03$ and compute the surface brightness of OviI emission following the procedure of Yoshikawa et al. (2004). We adopt a simple relation between the gas metallicity and the local gas density of the form $Z=0.02\left(\rho_{\text {gas }} / \bar{\rho}\right)^{0.3} \mathrm{Z}_{\odot}$, where $\bar{\rho}$ is the mean baryon density. We then compute the surface brightness for both $T_{\mathrm{e}}$ and $\bar{T}$. Figure 5 shows the

\footnotetext{
${ }^{4}$ The assumption of collisional ionization equilibrium may not be strictly valid in the WHIM. We nevertheless adopt this commonly-made assumption to compute the line emissivities in a post-processing manner.
} 


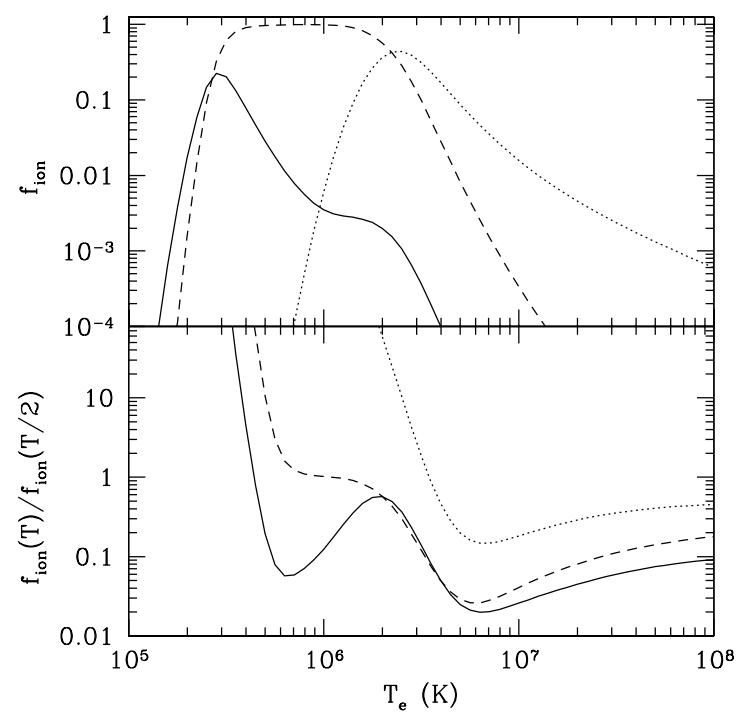

FIG. 4.- Relative abundance of oxygen ions $f_{\mathrm{O}_{\mathrm{VI}}}$ (solid), $f_{\mathrm{O}_{\mathrm{VII}}}$ (dashed), and $f_{\mathrm{O}_{\mathrm{VIII}}}$ (dotted) as a function of electron temperature. The bottom panel shows the ratio $f(T) / f(T / 2)$ which indicates the error induced by a factor of two shift (misestimate) in $T_{e}$.

derived profiles. While the overall results appear similar in the two cases, there are substantial local deviations. In particular, around some bright spots, the emissivity is greater by up to an order of magnitude when computed using the electron temperature. The ratio of the surface brightness $S\left(T_{\mathrm{e}}\right) / S(\bar{T})$ is shown in the bottom panel of Figure 5. There, we mark the regions with $S\left(T_{\mathrm{e}}\right)>10^{-11} \mathrm{ergs}^{-1} \mathrm{~cm}^{-2} \mathrm{sr}^{-1}$, which is the nominal detection limit of the proposed DIOS mission (Yoshikawa et al. 2003).

Similar considerations may also apply to efforts to study the WHIM using UV observations (e.g., Furlanetto et al. 2004 ), although the steep temperature dependence of $t_{\mathrm{ei}}$ implies that equipartition is more accurate in this regime (see Fig. 2). In principle, one can measure the degree of equilibration using various line intensities from far UV to soft X-rays (e.g., Ghavamian et al. 2001; Raymond et al. 2003) as well as the metal line Doppler widths. Measuring the age of the WHIM from the degree of equilibration will put a stringent constraint on theoretical models of largescale baryonic structure formation.
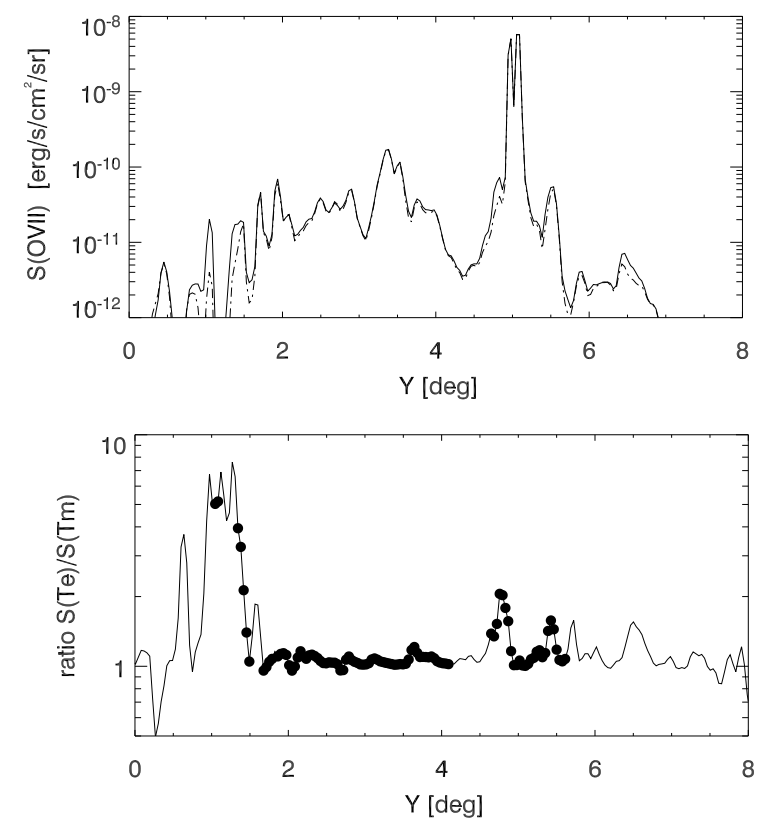

FIG. 5.- Surface brightness of OvII emission for the region indicated in the middle panel of Figure 1 using $T_{e}$ (solid line) and $\bar{T}$ (dot-dashed line). Bottom panel shows the ratio $S\left(T_{e}\right) / S(\bar{T})$. Dots in the bottom panel indicate the region where $S\left(T_{e}\right)$ is greater than $10^{-11} \mathrm{ergs}^{-1} \mathrm{~cm}^{-2} \mathrm{sr}^{-1}$.

Although our simulation does not include radiative cooling, we note that the lower electron temperature can have a significant impact on the radiative cooling efficiency in dense regions. The gas cooling rate has a very steep temperature dependence at $10^{5}<T<10^{6} \mathrm{~K}$ regardless of the metallicity (Sutherland \& Dopita 1993). As shown in Figure 2, recently shock-heated gas with $T \sim 10^{6} \mathrm{~K}$ may have an electron temperature $T_{\mathrm{e}} \sim 10^{5} \mathrm{~K}$. Such gas should cool rapidly and in a highly non-equilibrium manner. This warrants further study of the effect of the two-temperature structure on the IGM using a simulation with radiative cooling.

Overall, our simulation suggests that theoretical studies of the WHIM and correct interpretation of the observational data from current and future X-ray missions requires explicit consideration of these relaxation processes.

We thank Kohji Yoshikawa for providing emissivity data. The simulations were performed at the Center for Parallel Astrophysical Computing at the HarvardSmithsonian Center for Astrophysics.

\section{REFERENCES}

Cargill, P. J. \& Papadopoulos, K. 1988, ApJ, 329, L29

Cen, R. \& Ostriker, J. P. 1999, ApJ, 519, L109

Chieze, J.-P., Alimi, J.-M., \& Teyssier, R. 1998, ApJ, 495, 630

Courty, S. \& Alimi, J. M. 2004, A\&A, 416, 875

Croft, R.A.C. et al. 2002, ApJ, 581, 20

Davé, R., Cen, R., Ostriker, J. P., Bryan, G. L., Hernquist, L., Katz, N., Weinberg, D. H., Norman, M. L., \& O'Shea, B. 2001, ApJ, 552, 473

Fang, T. et al. 2004, ApJ in press

Finoguenov, A., Briel, U. G., \& Henry, J. P. 2003, A\&A, 410, 777

Fox, D. C. \& Loeb, A. 1997, ApJ, 491,459

Fukugita, M., Hogan, C. J. \& Peebles, P. J. E. 1998, 503, 518

Furlanetto, S.R., Schaye, J., Springel, V. \& Hernquist. L. 2003, ApJ, 599, L1

Furlanetto, S.R., Schaye, J., Springel, V. \& Hernquist. L. 2004, ApJ, 606, 221

Ghavamian, P. et al. 2001, ApJ, 547, 995
Keshet, U., Waxman, E., Loeb, A., Springel, V. \& Hernquist, L. 2003, ApJ, 585, 128

Laming, J. M. 2000, ApJS, 127, 409

Majumdar, S. \& Mohr, J. J. 2004, ApJ, 613, 41

Nicastro, F. et al. 2002, ApJ, 573, 157

Persic, M. \& Salucci, P. 1992, MNRAS, 258, 14

Raymond, J. C., et al. 2003, ApJ, 584, 770

Spergel, D.N., et al., 2003, ApJS, 148, 175

Spitzer, L., Jr. 1962, Physics of Fully Ionized Gases (New York: Wiley)

Springel, V. 2004, in preparation

Springel, V. \& Hernquist, L. 2002, MNRAS, 333, 649

Sutherland, R. \& Dopita, M. 1993, ApJS, 88, 253

Takizawa, M. 1998, ApJ, 509, 579

Yoshikawa, K. et al. 2003, PASJ, 55, 879

Yoshikawa, K. et al. 2004, PASJ in press astro-ph/0408140 